\title{
Characteristics of Accelerated Hand Osteoarthritis: Data from the Osteoarthritis Initiative
}

\author{
Julie E. Davis (D), Lena F. Schaefer, Timothy E. McAlindon, Charles B. Eaton, Mary B. Roberts, \\ Ida K. Haugen, Stacy E. Smith, Jeffrey Duryea, Bing Lu, and Jeffrey B. Driban 1
}

ABSTRACT. Objective. We aimed to determine whether hand joints develop an accelerated form of osteoarthritis (OA) and to characterize individuals who develop accelerated hand osteoarthritis (AHOA).

Methods. We evaluated 3519 participants in the Osteoarthritis Initiative with complete data for baseline and 48-month radiographic hand osteoarthritis (HOA). One reader scored posteroanterior radiographs of the dominant hand using a modified Kellgren-Lawrence (KL) scale and another reader scored the presence of central or marginal erosions. A third reader read images flagged for signs of diseases other than OA. We defined AHOA as $\geq 1$ joints that progressed from a KL grade of 0 or 1 at baseline to KL grade 3 or 4 at 48 months.

Results. The definition of AHOA was met by $1 \%$ over 4 years: 37 hands had 1 joint affected and 1 hand had 2 joints affected. At baseline, adults who developed AHOA were more likely to have hand pain (37\% vs 22\%), radiographic HOA (71\% vs 36\%), as well as central (22\% vs $7 \%$ ) and marginal erosions (11\% vs $2 \%$ ) in other joints compared to those without AHOA. Adults with AHOA were more likely to develop new erosions over 48 months (central 35\%, marginal 5\%) than those without AHOA (central 5\%, marginal 1\%). The most common locations of accelerated OA were the second metacarpophalangeal and first carpometacarpal joint.

Conclusion. Accelerated OA can occur in the hand, especially among digits commonly used for pinching and fine motor skills. (First Release December 1 2018; J Rheumatol 2019;46:422-8; doi:10.3899/jrheum.180240)

Key Indexing Terms: OSTEOARTHRITIS

HAND

EPIDEMIOLOGY

From the Division of Rheumatology, Tufts Medical Center; Department of Radiology, Brigham and Women's Hospital and Harvard Medical School; Division of Rheumatology, Immunology and Allergy, Brigham and Women's Hospital and Harvard Medical School, Boston, Massachusetts; Center for Primary Care and Prevention, Alpert Medical School of Brown University, Pawtucket, Rhode Island, USA; Department of Rheumatology, Diakonhjemmet Hospital, Oslo, Norway.

These analyses were financially supported by grants from the US National Institute of Arthritis and Musculoskeletal and Skin Diseases of the National Institutes of Health under Award Number R01-AR065977 and Award Number R01-AR066378. This work was also supported in part by the Houston Veterans Affairs Health Services Research and Development Center of Excellence (HFP90-020). The views expressed in this article are those of the authors and do not necessarily represent the views of the Department of Veterans Affairs.

J.E. Davis, BS, Research Assistant II, Division of Rheumatology, Tufts Medical Center; L.F. Schaefer, MD, Radiology Fellow, Department of Radiology, Brigham and Women's Hospital and Harvard Medical School; T.E. McAlindon, MD, MPH, Chief of Rheumatology, Division of Rheumatology, Tufts Medical Center; C.B. Eaton, MD, MS, Professor of Family Medicine and Epidemiology, Director of Center for Primary Care and Prevention, Alpert Medical School of Brown University; M.B. Roberts, $M S c$, Statistical Programmer, Center for Primary Care and Prevention, Alpert Medical School of Brown University; I.K. Haugen, MD, PhD, Rheumatology Fellow, Department of Rheumatology, Diakonhjemmet Hospital; S.E. Smith, MD, Section Head and Weissman Distinguished Chair of Musculoskeletal Radiology, Associate Radiology Residency Program Director, Department of Radiology, Brigham and Women's Hospital and Harvard Medical School; J. Duryea, PhD, Associate Research Associate, Associate Professor of Radiology, Department of Radiology, Brigham and Women's Hospital and Harvard Medical School; B. Lu, DrPH, MD, Assistant Professor of Medicine, Division of Rheumatology, Immunology and Allergy, Brigham and Women's Hospital and Harvard Medical School; J.B. Driban, PhD, ATC, CSCS, Associate Professor, Division of Rheumatology, Tufts Medical Center.

Address correspondence to J.B. Driban, Division of Rheumatology, Tufts Medical Center; 800 Washington St., Box 406, Boston, Massachusetts 02111,USA.E-mail: jeffrey.driban@tufts.edu

Accepted for publication August 15, 2018.

Hand osteoarthritis (HOA) is a painful and debilitating disease, as well as the most frequent location of $\mathrm{OA}^{1}$. There may be considerable heterogeneity within this condition, which previous investigators have tried to divide into subsets based on the presence of erosions or affected joints [e.g., distal interphalangeal (DIP) ${ }^{2}$, proximal interphalangeal (PIP), or thumb-base $]^{3,4,5}$.

Previous studies have analyzed incidence of $\mathrm{HOA}^{4,6,7,8,9}$, but few have assessed the incidence of HOA subsets ${ }^{4,6,10,11}$. For example, within the Osteoarthritis Initiative (OAI), 19\% of people developed radiographic OA in at least 1 DIP or PIP over 4 years, but only $3 \%$ developed erosive $\mathrm{OA}^{4}$. Assessing the onset of HOA subsets is challenging because there are few large cohorts with sufficient sample size to longitudinally analyze the potential subsets ${ }^{7}$.

A potentially understudied subset of HOA is individuals who experience a dramatic accelerated rate of $\mathrm{OA}$ onset. Larger joints such as the knee, hip, and less frequently shoulder may develop an accelerated form of $\mathrm{OA}^{12,13,14,15,16,17,18,19}$.

Personal non-commercial use only. The Journal of Rheumatology Copyright $\odot$ 2019. All rights reserved 
Adults who develop accelerated knee OA, in which they progress from normal radiographic appearance to advance-stage disease within 4 years and often in less than 12 months, report more pain and dysfunction than adults who develop a more gradual onset of $\mathrm{OA}^{2,20,21}$. It is unknown whether such a novel subset of OA exists among small joints in adults, particularly the joints of the hand. Therefore, we aimed to determine whether hand joints develop an accelerated form of OA, to characterize individuals who develop accelerated HOA (AHOA), and to compare them to those who do not. We anticipate that those with AHOA will have more erosions, particularly central erosions, than those without AHOA because central erosions are a risk factor for cartilage $\operatorname{loss}^{22}$.

\section{MATERIALS AND METHODS}

Study design. To characterize AHOA, we identified individuals using data from baseline and the 48-month followup visit of the OAI. The OAI is a multicenter cohort study of 4796 adults with or at risk for symptomatic knee OA. Four clinical sites (Memorial Hospital of Rhode Island, Ohio State University, the University of Maryland and Johns Hopkins University, and the University of Pittsburgh) recruited participants between February 2004 and May 2006. OAI data and protocols are available for free public access ${ }^{23}$. Institutional review boards at each OAI clinical site and the OAI coordinating center (University of California, San Francisco, California, USA) approved the OAI study. All participants provided informed consent prior to participation.

Participant selection. We initially evaluated 3616 participants in the OAI with good-quality radiographs of the dominant hand at the baseline and 48-month visits. We excluded 19 people who had radiographic evidence suggestive of a musculoskeletal pathology other than OA [e.g., psoriatic arthritis, rheumatoid arthritis (RA), osteitis, gout, ankylosis; $n=3597]$. These findings were initially identified by 1 reader (IKH) and confirmed by a musculoskeletal radiologist (SES). To minimize misclassification, we also excluded 78 people because a reader was unable to assess radiographic severity [Kellgren-Lawrence (KL) grade] at 16 joints (i.e., 12 finger joints, 4 thumb joints) because of hand positioning $(\mathrm{n}=3519)$.

Dominant hand. The OAI staff asked study participants if they were right- or left-handed and $95 \%$ of participants responded with left or right. If a participant answered ambidextrous or unknown or if data were missing, then we used the following standardized rules to define the dominant hand: (1) if the person had unilateral hand radiographs, then we selected the imaged hand (4\%); and (2) if the person had bilateral hand radiographs, we selected the dominant hand based on the ipsilateral hand to the foot a participant reported using to kick a ball (1\%).

Hand radiographs. Staff at each OAI site collected posteroanterior radiographs of the dominant hand of each participant at baseline and 48 months. The positioning of the hand required the participant to be placed with the elbow flexed $90^{\circ}$ and the forearm flat against the table. While most participants had unilateral hand radiographs, only $22 \%$ had bilateral images; hence, our work focused on the dominant hand.

Hand radiographic readings. Using the radiographs, readers scored 16 joints of the dominant hand: second to fifth DIP joints, second to fifth PIP joints, first to fifth metacarpophalangeal (MCP) joints, thumb interphalangeal (IP) joint, and the thumb-base joints [first carpometacarpal (CMC) joint and scaphotrapezial (STT) joint ${ }^{11}$. Specifically, a radiology fellow (LFS), who was trained for this project by a board-certified musculoskeletal radiologist (SES), scored radiographic severity using a modified KL scale. A software displayed baseline and followup images side by side but blinded the reader to time. The modified KL scale was used in the Framingham Study ${ }^{6}$ KL $0=$ no $\mathrm{OA}[$ no osteophyte or joint space narrowing (JSN)], KL 1 = questionable osteophyte or JSN, KL 2 = small osteophyte(s) or mild JSN,
KL 3 = moderate osteophyte(s) or JSN, KL 4 = large osteophyte(s) or JSN. LFS scored 100 randomly selected pairs of hand radiographs twice, with good intrareader agreement (weighted $x>0.84$ across 16 joints).

Another reader (IKH) scored the presence of central or marginal erosions ( $x>0.79$ across joints from 70 hands). The presence of central erosions was reported according to the Osteoarthritis Research Society International atlas $^{24}$. The presence of marginal erosions was reported based on the Sharp-van der Heijde method ${ }^{25}$. However, in contrast to this method, we also scored the DIP joints, because they are frequently affected in HOA, and only the thumb-base joints were scored in the wrist. Central and marginal erosions were scored because prior reports indicate that both may be identified among people with $\mathrm{HOA}^{10,26,27,28}$.

Definition of $A H O A$. We defined incident $\mathrm{AHOA}$ as a hand with at least 1 joint that progressed from no radiographic $\mathrm{OA}(\mathrm{KL}=0$ or 1$)$ at baseline to advance-stage disease at 48 months $(K L=3$ or 4$)$. A strength of this definition is that it typically requires a change in osteophytes and joint space. We previously validated this definition for accelerated knee $\mathrm{OA}^{21}$.

Avoiding misclassification. Readings from the radiology fellow were sent to a rheumatologist with an expertise in HOA epidemiology (IKH). The rheumatologist added scores for specific joint features (e.g., presence of central erosions) and flagged hands when she had concerns about pathology unrelated to OA. The flagged hands were then sent to the board-certified musculoskeletal radiologist, who reviewed the images and offered a final opinion on whether the person should be excluded because of radiographic evidence of other pathology. Finally, a rheumatologist (TEM) reviewed medication use among participants with possible AHOA to determine whether anyone should be excluded based on indications of treatment for an inflammatory condition.

Participant characteristics. We selected demographic, anthropometric, and additional participant characteristics. These were acquired based on a standard protocol ${ }^{23}$. Specifically, we extracted several key baseline variables: age, body mass index (BMI), sex, and radiographic status. We also derived a person's baseline menopause status and the presence of metabolic syndrome using publicly available data. Menopausal status at baseline was based on a woman's response to "When was your last natural menstrual period? Do not include bleeding due to taking female hormone pills or patches." Based on this question, we classified each woman into one of 3 states: (1) premenopause if she indicated that she had a natural menstrual period "within the past 12 months," (2) perimenopause if she indicated that she had a natural menstrual period in " 1 to 2 years ago" or " 3 to 4 years ago," and (3) postmenopause if she answered " 5 or more years ago." We excluded women who did not know when their last natural menstrual period occurred. If a woman left this question unanswered and she reported a history of a hysterectomy, then we considered her in perimenopause if the hysterectomy was in the 5 years prior to OAI baseline and postmenopause if the hysterectomy was reported $\geq 5$ years prior to the baseline visit. The presence of metabolic syndrome was defined by having at least 2 of the 4 following criteria: (1) self-reported diabetes on the Charlson Comorbidity Index, (2) use of cholesterol medication (derived from a medication inventory form), (3) high blood pressure (systolic pressure $\geq 130$ or diastolic pressure $\geq 85$ ) or use of hypertension medication (derived from a medication inventory form), and (4) central adiposity based on sex-specific cutoffs for weight circumference. Additionally, participants were asked at baseline about hand pain: "During the past 30 days, which of these joints have had pain, aching, or stiffness on most days? By most days, we mean more than half the days of a month." Participants would then indicate left or right hand ${ }^{23}$. The baseline variables were extracted from the allclinical00 (version 0.2.2) and mif00 (version 0.2.2) files, which are available on the OAI Website.

At each OAI followup visit, the OAI staff asked participants if a doctor said they broke or fractured hand bone(s) since their last visit about 12 months prior. The participant then answered yes or no [files: allclinical01 (version 1.2.1), allclinical03 (version 3.2.1), allclinical05 (version 5.2.1), allclinical06 (version 6.2.1)].

Statistical methods. We calculated descriptive statistics to characterize

Personal non-commercial use only. The Journal of Rheumatology Copyright (C) 2019. All rights reserved. 
people with and without AHOA. For categorical variables, we calculated the differences in frequency between those with and without AHOA and the Wald (Asymptotic) 95\% CI. For continuous measures, we calculated the mean differences and the $95 \%$ CI based on pooled and Satterthwaite $95 \%$ intervals depending on whether variances were equal between groups, respectively. We conducted an investigative logistic regression to test the association between incident AHOA and 5 baseline characteristics in 1 model (i.e., sex, presence of radiographic HOA, presence of central erosions, body weight, and age). As a sensitivity analysis, we calculated the descriptive characteristics for people with and without AHOA among those who had no joints with advance-stage disease in the dominant hand at baseline (KL $=3$ or 4). Results were generated using SAS Enterprise Guide 7.1.

Ethical standards. The OAI was approved and meets all criteria for ethical standards regarding human and animal studies defined in the 1964 Declaration of Helsinki and all amendments made after. Institutional review boards at each OAI clinical site and the OAI coordinating center (University of California, San Francisco, California, USA) approved the OAI study (approval number 10-00532). All participants provided informed consent prior to participation.

\section{RESULTS}

We analyzed data from 3519 participants. There were no meaningful differences between OAI participants included $(n=3519)$ or excluded $(n=1277)$ from these analyses for age (included: $60.8 \pm 9.2$ yrs vs excluded: $62.2 \pm 9.0 \mathrm{yrs}$ ), BMI $\left(28.7 \pm 4.9 \mathrm{~kg} / \mathrm{m}^{2}\right.$ vs $\left.28.4 \pm 4.8 \mathrm{~kg} / \mathrm{m}^{2}\right)$, or proportion of females (58\% vs $60 \%)$, presence of hand pain $(28 \%$ vs $29 \%$ ), or self-reported diagnosis of HOA (17\% vs $16 \%)$.
The definition of AHOA was met by $1 \%(95 \% \mathrm{CI}$ $0.8-1.5 \%$ ) over 4 years: 37 hands had 1 joint affected and 1 hand had 2 joints affected.

At baseline, the AHOA group was slightly more female (74\% vs 57\%), older (64.9 vs $61.0 \mathrm{yrs)}$, and more likely to have hand pain $(37 \%$ vs $22 \%$; Table 1$)$. Only 1 person $(3 \%)$ with AHOA had a fracture during the observation period, compared to 56 people (2\%) with fractures in the control group. Additionally, $8 \%$ of adults with AHOA also developed accelerated knee OA, compared with $3 \%$ of adults without AHOA. When we calculated the descriptive characteristics among adults without advance-stage disease in the dominant hand at baseline, we found similar results (Table 2).

Location of AHOA. Over 48 months, the most common locations of AHOA were the thumb and second digit (Figure 1). The most commonly affected joints were the MCP2 and CMC1, which each had 10 cases.

Baseline radiographic characteristics. At baseline, adults who developed AHOA were more likely to have radiographic $\operatorname{HOA}(\mathrm{KL} \geq 2)$ in at least 1 other joint $(71 \%$ vs $35 \%)$, at least 1 joint with central erosions ( $22 \%$ vs $7 \%)$, and at least 1 joint with marginal erosions ( $11 \%$ vs $2 \%$ ) compared with those who did not develop AHOA (Table 1). Most erosions were at joints other than the joint that developed AHOA (Table 3), with only 1 person having a marginal erosion at a joint that

Table 1. Characteristics of adults who develop accelerated HOA. Values are $\mathrm{n}(\%)$ unless otherwise specified.

\begin{tabular}{|c|c|c|c|}
\hline Variables & No Accelerated HOA, $\mathrm{n}=3481$ & Accelerated $\mathrm{HOA}, \mathrm{n}=38$ & Difference Between Groups (95\% CI) \\
\hline \multicolumn{4}{|l|}{ Baseline characteristics* } \\
\hline Female & $1993(57)$ & $28(74)$ & $16(2-31)^{\mathrm{a}}$ \\
\hline Perimenopause & $274(14)$ & $2(7)$ & $-7(-16$ to 3$)$ \\
\hline Postmenopause & $1411(71)$ & $24(86)$ & $15(1-28)$ \\
\hline Metabolic syndrome, 2-4 components & $1914(57)$ & $23(61)$ & $3(-13 \text { to } 19)^{\mathrm{a}}$ \\
\hline High blood pressure & $2079(59)$ & $24(63)$ & $4(-12$ to 19$)$ \\
\hline Large waist circumference & $2540(75)$ & $29(76)$ & $2(-12 \text { to } 15)^{\mathrm{a}}$ \\
\hline Physician-diagnosed HOA & $534(15)$ & $16(42)$ & $27(11-43)$ \\
\hline Prevalent hand pain & $756(22)$ & $14(37)$ & $15(0-31)$ \\
\hline Age, yrs, mean (SD) & $61.0(9.1)$ & $64.9(8.3)$ & $3.9(1.0-6.8)$ \\
\hline Body mass index, $\mathrm{kg} / \mathrm{m}^{2}$, mean (SD) & $28.5(4.8)$ & $27.4(4.5)$ & $-1.1(-2.7$ to 0.4$)$ \\
\hline Marginal erosions, $\geq 1$ joint & $81(2)$ & $4(11)$ & $8(-2 \text { to } 18)^{\mathrm{a}}$ \\
\hline No. joints with $\mathrm{KL} \geq 3$, mean (SD) & $0.5(1.2)$ & $1.1(2.0)$ & $0.6(-0.02$ to 1.3$)$ \\
\hline \multicolumn{4}{|l|}{ Incident findings over time } \\
\hline Incident accelerated knee OA & $105(3)$ & $3(8)$ & $5(-4$ to 13$)$ \\
\hline New central erosions over 48 mos & $158(5)$ & $13(35)$ & $31(15-46)^{\mathrm{a}}$ \\
\hline New marginal erosions over 48 mos & $50(1)$ & $2(5)$ & $4(-3$ to 11$)$ \\
\hline
\end{tabular}

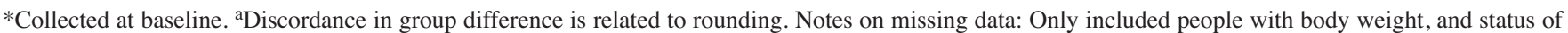
self-reported hand pain (yes or no), and physician-diagnosed HOA (yes and no or unknown) at baseline. Missing data: Less than $3 \%$ missing data for menopause status, central erosions, marginal erosions, diabetes status, and waist circumference. Metabolic syndrome (n missing $=137$ controls). No other missing data. HOA: hand osteoarthritis; KL: Kellgren-Lawrence arthritis grading scale. 
Table 2. Characteristics of adults who develop accelerated HOA with no joints with advance-stage disease (Kellgren-Lawrence grade 3 or 4) at baseline. Values are $\mathrm{n}(\%)$ unless otherwise specified.

\begin{tabular}{|c|c|c|c|}
\hline Variables & No Accelerated HOA, $\mathrm{n}=2741$ & Accelerated HOA, $\mathrm{n}=21$ & Difference Between Groups (95\% CI) \\
\hline \multicolumn{4}{|l|}{ Baseline characteristics* } \\
\hline Female & $1495(55)$ & $16(76)$ & $22(3-40)^{\mathrm{a}}$ \\
\hline Perimenopause & $250(17)$ & $1(6)$ & $-11(-23$ to 1$)$ \\
\hline Postmenopause & $947(64)$ & $13(81)$ & $18(-2 \text { to } 37)^{\mathrm{a}}$ \\
\hline Metabolic syndrome, 2-4 components & $1369(54)$ & $10(50)$ & $-4(-26$ to 18$)$ \\
\hline High blood pressure & $1492(56)$ & $13(65)$ & $9(-12$ to 30$)$ \\
\hline Large waist circumference & $1877(73)$ & $13(65)$ & $-8(-29$ to 13$)$ \\
\hline Physician-diagnosed HOA & $285(10)$ & $5(24)$ & $13(-5 \text { to } 32)^{\mathrm{a}}$ \\
\hline Prevalent hand pain & $470(17)$ & $9(43)$ & $26(5-47)^{\mathrm{a}}$ \\
\hline Age, yrs, mean (SD) & $59.1(8.6)$ & $62.4(8.0)$ & $3.3(0.43-7.0)$ \\
\hline Body mass index, $\mathrm{kg} / \mathrm{m}^{2}$, mean (SD) & $28.6(4.9)$ & $27.4(4.8)$ & $-1.2(-3.3$ to 0.9$)$ \\
\hline Marginal erosions, $\geq 1$ joint & $51(2)$ & $1(5)$ & $3(-6 \text { to } 13)^{\mathrm{a}}$ \\
\hline \multicolumn{4}{|l|}{ Incident findings over time } \\
\hline Incident accelerated knee OA & $78(3)$ & $1(5)$ & $2(-7$ to 11$)$ \\
\hline New central erosions over 48 mos & $24(<1)$ & $6(30)$ & $29(9-49)$ \\
\hline New marginal erosions over 48 mos & $28(1)$ & $1(5)$ & $4(-6$ to 14$)$ \\
\hline
\end{tabular}

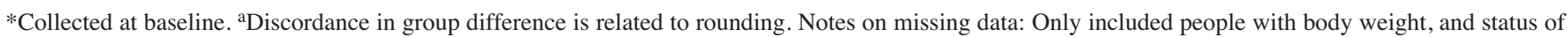
self-reported hand pain (yes or no), and physician-diagnosed HOA (yes and no or unknown) at baseline. Missing data: Less than 3\% missing data for menopause status, central erosions, marginal erosions, diabetes status, and waist circumference. Metabolic syndrome (n missing $=137$ controls). No other missing data. HOA: hand osteoarthritis.

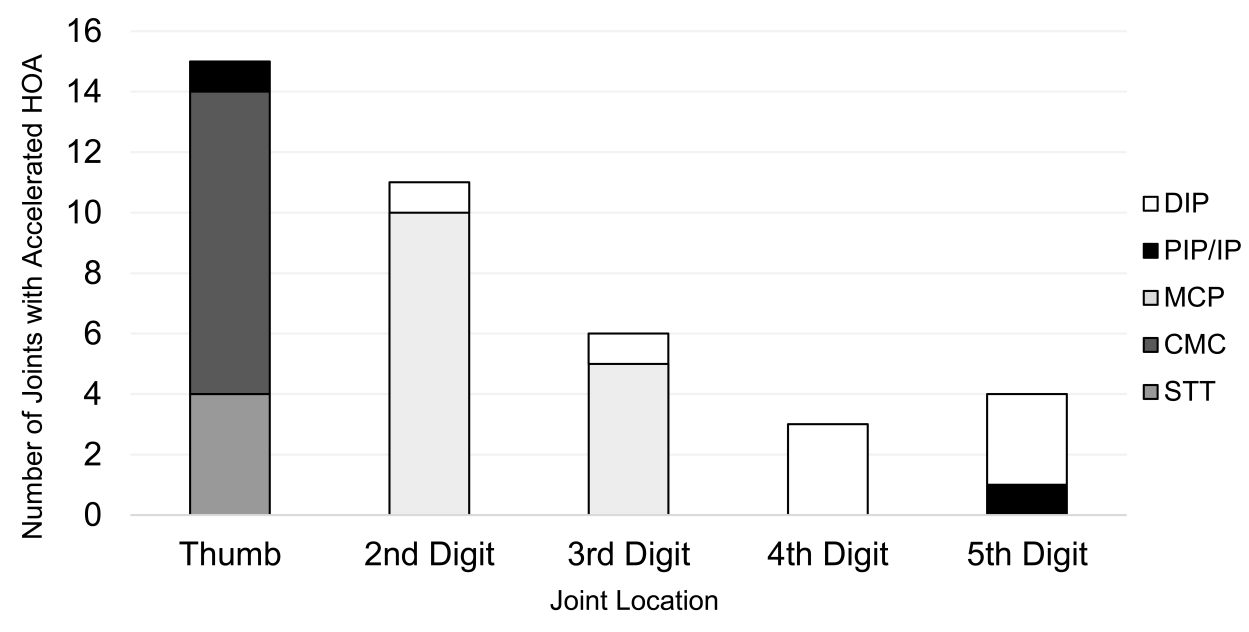

Figure 1. Patterns of accelerated HOA by location. HOA: hand osteoarthritis; DIP: distal interphalangeal; PIP/IP: proximal interphalangeal; MCP: metacarpophalangeal; CMC: carpometacarpal; STT: scaphotrapezial.

developed AHOA (MCP2). Overall, these results were similar among adults with no advance-stage disease in the dominant hand at baseline; except for no group difference for the presence of baseline central erosions ( $0 \%$ vs $<1 \%$; Table 2$)$. Investigative multivariable analysis of baseline characteristics. Our investigative analysis was 1 model that included
5 baseline characteristics: sex, presence of radiographic HOA, presence of central erosions, body weight, and age. We found that only the presence of radiographic HOA was associated with incident AHOA (OR 4.3, 95\% CI 1.8-10.3, $\mathrm{p}=0.001)$. The other variables had $\mathrm{p}$ values $>0.40$; except weight $(\mathrm{p}=0.09)$.

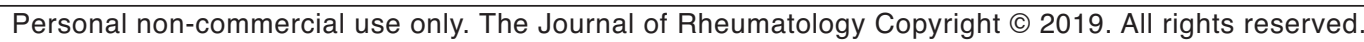


Table 3. Type and location of erosions among adults with accelerated HOA $(\mathrm{n}=38)$. Values are $\mathrm{n}$.

\begin{tabular}{lcc}
\hline Variables & $\begin{array}{c}\text { Hands with Erosion at Joint } \\
\text { with Accelerated HOA }\end{array}$ & $\begin{array}{c}\text { Hands with Erosion at a Joint } \\
\text { without Accelerated HOA }\end{array}$ \\
\hline Baseline erosion & & 8 \\
$\quad$ Central erosion & 0 & 3 \\
$\quad$ Marginal erosion & 1 & 9 \\
New erosion over 48 mos & 7 & 1 \\
$\quad$ Central erosion & 1 & \\
$\quad$ Marginal erosion & 1 & \\
\hline
\end{tabular}

Note: Six people had both baseline erosions and new erosions. HOA: hand osteoarthritis.

Radiographic characteristics over time. Adults with AHOA were more likely to develop new erosions in either the same or another joint over 48 months (central 35\%, marginal 5\%) than those who did not develop AHOA (central 5\%, marginal $1 \%$ ). These results were similar among adults without advance-stage disease in the dominant hand at baseline (Table $2)$. Eight people $(21 \%)$ who developed AHOA had an incident erosion in the same joint that worsened, with only 1 of them being marginal and the remaining 7 being a central erosion. Ten people (26\%) with AHOA developed an incident erosion at a joint other than the one with accelerated progression. Similarly, only 1 of those was marginal and 9 were central (Table 3).

\section{DISCUSSION}

We identified and characterized AHOA and found that the affected joints are most common in the thumb-base and second MCP joints, which are commonly used for fine motor skills such as a pincer grasp. Further, people with radiographic OA in other hand joints, particularly with erosions, were more likely to develop AHOA. Hence, AHOA may be a subset of HOA that is associated with erosive HOA. This supports the notion that erosive HOA is a more rapidly progressing phenotype.

We found that AHOA manifested overwhelmingly at $\mathrm{CMC} 1$ and $\mathrm{MCP} 2$. While $\mathrm{OA}$ at $\mathrm{CMC} 1$ is fairly common ${ }^{5}$, the prevalence of MCP OA is low and more common among men $(11.9 \% \text { in men, } 6.8 \% \text { in women })^{6}$. The few investigators who examined $\mathrm{OA}$ in MCP joints found that when these joints are affected, the prevalence is higher at MCP1, MCP2, and $\mathrm{MCP} 3^{6,10,29}$. OA of MCP joints may be associated with heavy labor because forces are higher at these joints than the more distal ones (DIP or PIP joints) $)^{6,30,31}$. With the high occurrence of AHOA at MCP2 and the thumb, both involved in gripping, it is possible that individuals involved in manual labor may be more at risk for accelerated $\mathrm{OA}$ at these proximal joints because stresses are higher than at distal joints. Future studies with occupational and hand function data should test these claims, as well as investigate how much of MCP OA may be accelerated OA.

We detected a relationship between hand erosions and AHOA, although they often occurred in different joints within the hand. The onset of hand erosions or AHOA are more common among those with radiographic HOA at baseline compared with those without $\mathrm{HOA}^{22,28}$. When we excluded people with advance-stage disease at baseline, we still observed a possible relationship between incident AHOA and new central erosions, despite the absence of a relationship between AHOA and baseline central erosions. These seemingly contradictory findings may be explained by a bias introduced in the cross-sectional analysis by excluding those with advanced-stage disease at baseline.

Erosions are typically found among those with inflammatory conditions such as RA, so finding them frequently in AHOA was particularly interesting ${ }^{3,22}$. Erosions at MCP joints would suggest an inflammatory condition; however, only 1 person had an erosion at an MCP joint. The rest of the erosions were heavily weighted toward the IP joints, with a few at the CMC and STT joints, which is consistent with HOA.

It would be particularly interesting to examine the potential differences between the involvement of central versus marginal erosions, especially because central erosions are a risk factor for cartilage loss ${ }^{27}$. Previous magnetic resonance imaging studies have suggested that marginal erosions may be more common in HOA than we have been able to demonstrate using conventional radiographs ${ }^{32,33,34}$. However, we acknowledge that the clinical importance is unknown, because few studies have investigated the prevalence and involvement of marginal erosions in HOA. Marginal erosions are typical among other rheumatic diseases, such as RA. However, 1 or few marginal erosions are unspecific and do not necessarily indicate that an individual has an inflammatory joint disease.

The relationship between erosive HOA and AHOA should be further analyzed because they may share common causes or risk factors or be closely related subsets ${ }^{35}$. For example, HOA, particularly erosive HOA, has been associated with reduced bone mineral content and density in the hand and wrist $^{36,37,38}$. This is relevant owing to prior studies associating accelerated $\mathrm{OA}$ at large joints with significant bone damage ${ }^{19,39}$.

There were slight differences in our demographics and participant characteristics. We found that people with AHOA

Personal non-commercial use only. The Journal of Rheumatology Copyright @ 2019. All rights reserved. 
tended to be female, slightly older, and more likely to report pain. Certain phenotypes within HOA differ between sexes (e.g., joint location, erosive OA $)^{6}$. Our findings complement a prior study in which the investigators found that erosive HOA, which may be related to AHOA, was mainly found among women ${ }^{6}$. Additionally, those with AHOA were more likely to report hand pain at baseline than those without AHOA. This is consistent with our findings that accelerated knee OA is also often preceded by more knee pain than common knee OA and that pain may precede accelerated structural changes ${ }^{2,11,20}$

While our study identified accelerated OA in hands, we acknowledge that there are limitations. With a larger sample size, it could be possible to analyze more complex interactions between risk factors. Even with a smaller sample size of cases, we were able to identify potentially important characteristics and patterns of those with AHOA. Additionally, without extensive laboratory work, we depended solely on radiographs and medication use to eliminate other types of arthritis, so it is possible that some people may have still been misclassified despite our best effort. Another limitation was our reliance on dominant hand radiographs because bilateral images were only collected on $\sim 20 \%$ of participants. Prior investigators suggested that nondominant thumb-base OA may be more common than in the dominant hand ${ }^{5,6}$. It would be beneficial to have bilateral hand radiographs to determine whether accelerated $\mathrm{OA}$ at $\mathrm{CMC1}$ is more prevalent than we found. The lack of radiographs for the nondominant hand does not alter our finding that accelerated OA occurs in hands and warrants further study. We may also be limited by defining AHOA based on disease onset at just 1 joint. By including hands with only 1 affected joint, we may be including joints that develop accelerated OA because of trauma; however, this was the goal because accelerated OA at the knee is related to joint trauma ${ }^{12}$. Future research will be needed to determine whether accelerated OA in the hand is also associated with joint trauma.

We have also been cautious to minimize reference to incident accelerated OA because we are unable to rule out that some joints had accelerated OA prior to the OAI, having entered the study with a KL grade of 3 or 4 in $\geq 1$ joint. In the current cohort, it would be infeasible because of the small number of cases to select the few hands in which no joint has radiographic $\mathrm{OA}$ at baseline. Further, baseline radiographic HOA is associated with accelerated OA. If we exclude people with radiographic $\mathrm{HOA}$ at baseline, we may introduce a selection bias. Hence, it may be valuable to further investigate AHOA among people under 45 years of age. Finally, the OAI is a rich dataset of individuals with or at-risk for symptomatic knee OA, hence it is not a population-based study. Therefore, the incidence over 4 years and the observed associations may not reflect the general population and future studies may be warranted to determine how the estimated proportions of person with AHOA and associations differ between the OAI and population-based cohorts. Despite this limitation, the OAI is a large dataset, which enabled us to confirm the presence of accelerated OA in the hand and raise awareness about this novel subset of people with AHOA.

We identified an accelerated form of OA in hand joints. AHOA may be a unique phenotype in which individuals are more likely to progress at the MCP 2 and $\mathrm{CMC} 1$ joints. Those with AHOA may be more likely to have radiographic HOA. Our results identify an underreported phenotype of HOA that presents with radiographic differences and a worrisome acceleration of HOA.

\section{REFERENCES}

1. Kalichman L, Cohen Z, Kobyliansky E, Livshits G. Patterns of joint distribution in hand osteoarthritis: contribution of age, sex, and handedness. Am J Hum Biol 2004;16:125-34.

2. Driban JB, Price LL, Eaton CB, Lu B, Lo GH, Lapane KL, et al. Individuals with incident accelerated knee osteoarthritis have greater pain than those with common knee osteoarthritis progression: data from the Osteoarthritis Initiative. Clin Rheumatol 2016;35:1565-71.

3. Gazeley DJ, Yeturi S, Patel PJ, Rosenthal AK. Erosive osteoarthritis: a systematic analysis of definitions used in the literature. Semin Arthritis Rheum 2017;46:395-403.

4. Haugen IK, Magnusson K, Turkiewicz A, Englund M. The prevalence, incidence, and progression of hand osteoarthritis in relation to body mass index, smoking, and alcohol consumption. J Rheumatol 2017;44:1402-9.

5. Wilder FV, Barrett JP, Farina EJ. Joint-specific prevalence of osteoarthritis of the hand. Osteoarthritis Cartilage 2006;14:953-7.

6. Haugen IK, Englund M, Aliabadi P, Niu J, Clancy M, Kvien TK, et al. Prevalence, incidence and progression of hand osteoarthritis in the general population: the Framingham Osteoarthritis Study. Ann Rheum Dis 2011;70:1581-6.

7. Chaisson CE, Zhang Y, McAlindon TE, Hannan MT, Aliabadi P, Naimark A, et al. Radiographic hand osteoarthritis: incidence, patterns, and influence of pre-existing disease in a population based sample. J Rheumatol 1997;24:1337-43.

8. Prieto-Alhambra D, Judge A, Javaid MK, Cooper C, Diez-Perez A, Arden NK. Incidence and risk factors for clinically diagnosed knee, hip and hand osteoarthritis: influences of age, gender and osteoarthritis affecting other joints. Ann Rheum Dis 2014; 73:1659-64.

9. Reyes C, Leyland KM, Peat G, Cooper C, Arden NK, Prieto-Alhambra D. Association between overweight and obesity and risk of clinically diagnosed knee, hip, and hand osteoarthritis: a population-based cohort study. Arthritis Rheumatol 2016; 68:1869-75.

10. Addimanda O, Cavallari C, Pignotti E, Pulsatelli L, Mancarella L, Ramonda R, et al. Radiographic involvement of metacarpophalangeal and radiocarpal joints in hand osteoarthritis. Clin Rheumatol 2017;36:1077-82.

11. Schaefer LF, McAlindon TE, Eaton CB, Roberts MB, Haugen IK, Smith SE, et al. The associations between radiographic hand osteoarthritis definitions and hand pain: data from the Osteoarthritis Initiative. Rheumatol Int 2017;38:403-13.

12. Driban JB, Eaton CB, Lo GH, Ward RJ, Lu B, McAlindon TE. Association of knee injuries with accelerated knee osteoarthritis progression: data from the Osteoarthritis Initiative. Arthritis Care Res 2014;66:1673-9.

13. Irwin LR, Roberts JA. Rapidly progressive osteoarthrosis of the hip. J Arthroplasty 1998;13:642-6.

Personal non-commercial use only. The Journal of Rheumatology Copyright @ 2019 . All rights reserved. 
14. Batra S, Batra M, McMurtrie A, Sinha A. Rapidly destructive osteoarthritis of the hip joint: a case series. J Orthop Surg Res 2008;3:3.

15. Boutry N, Paul C, Leroy X, Fredoux D, Migaud H, Cotten A. Rapidly destructive osteoarthritis of the hip: MR imaging findings. AJR Am J Roentgenol 2002;179:657-63.

16. Postel M, Kerboull M. Total prosthetic replacement in rapidly destructive arthrosis of the hip joint. Clin Orthop Relat Res 1970;72:138-44.

17. Della Torre P, Picuti G, Di Filippo P. Rapidly progressive osteoarthritis of the hip. Ital J Orthop Traumatol 1987;13:187-200

18. Yamamoto T, Bullough PG. The role of subchondral insufficiency fracture in rapid destruction of the hip joint: a preliminary report. Arthritis Rheum 2000;43:2423-7.

19. Hochberg MC. Serious joint-related adverse events in randomized controlled trials of anti-nerve growth factor monoclonal antibodies Osteoarthritis Cartilage 2015;23 Suppl 1:S18-S21.

20. Davis J, Eaton CB, Lo GH, Lu B, Price LL, McAlindon TE, et al. Knee symptoms among adults at risk for accelerated knee osteoarthritis: data from the Osteoarthritis Initiative. Clin Rheumatol 2017;36:1083-9.

21. Driban JB, Stout AC, Lo GH, Eaton CB, Price LL, Lu B, et al. Best performing definition of accelerated knee osteoarthritis: data from the Osteoarthritis Initiative. Ther Adv Musculoskelet Dis 2016;8:165-71

22. Schett $\mathrm{G}$, Gravallese E. Bone erosion in rheumatoid arthritis: mechanisms, diagnosis and treatment. Nat Rev Rheumatol 2012;8:656-64

23. The Osteoarthritis Initiative. [Internet. Accessed October 30, 2018.] Available from: http://oai.epi-ucsf.org

24. Altman RD, Gold GE. Atlas of individual radiographic features in osteoarthritis, revised. Osteoarthritis Cartilage 2007;15 Suppl A:A1-A56.

25. van der Heijde D. How to read radiographs according to the Sharp/van der Heijde method. J Rheumatol 2000;27:261-3.

26. Addimanda O, Mancarella L, Dolzani P, Punzi L, Fioravanti A, Pignotti E, et al. Clinical and radiographic distribution of structural damage in erosive and nonerosive hand osteoarthritis. Arthritis Care Res 2012;64:1046-53.

27. Marshall M, Peat G, Nicholls E, van der Windt D, Myers H, Dziedzic K. Subsets of symptomatic hand osteoarthritis in community-dwelling older adults in the United Kingdom: prevalence, inter-relationships, risk factor profiles and clinical characteristics at baseline and 3-years. Osteoarthritis Cartilage 2013;21:1674-84.
28. Marshall M, Nicholls E, Kwok WY, Peat G, Kloppenburg M, van der Windt D, et al. Erosive osteoarthritis: a more severe form of radiographic hand osteoarthritis rather than a distinct entity? Ann Rheum Dis 2015;74:136-41.

29. Gupta KB, Duryea J, Weissman BN. Radiographic evaluation of osteoarthritis. Radiol Clin North Am 2004;42:11-41.

30. Williams WV, Cope R, Gaunt WD, Adelstein EH, Hoyt TS, Singh A, et al. Metacarpophalangeal arthropathy associated with manual labor (Missouri metacarpal syndrome). Clinical radiographic, and pathologic characteristics of an unusual degeneration process. Arthritis Rheum 1987;30:1362-71.

31. Chaisson CE, Zhang Y, Sharma L, Felson DT. Higher grip strength increases the risk of incident radiographic osteoarthritis in proximal hand joints. Osteoarthritis Cartilage;8 Suppl A:S29-32.

32. Tan AL, Grainger AJ, Tanner SF, Shelley DM, Pease C, Emery P, et al. High-resolution magnetic resonance imaging for the assessment of hand osteoarthritis. Arthritis Rheum 2005;52:2355-65.

33. Haugen IK, Boyesen P, Slatkowsky-Christensen B, Sesseng S, Bijsterbosch J, van der Heijde D, et al. Comparison of features by MRI and radiographs of the interphalangeal finger joints in patients with hand osteoarthritis. Ann Rheum Dis 2012;71:345-50.

34. Haugen IK, Slatkowsky-Christensen B, Bøyesen P, Sesseng S, van der Heijde D, Kvien TK. MRI findings predict radiographic progression and development of erosions in hand osteoarthritis. Ann Rheum Dis 2016;75:117-23.

35. Boeters DM, Nieuwenhuis WP, van Steenbergen HW, Reijnierse M, Landewé RB, van der Helm-van Mil AHM. Are MRI-detected erosions specific for RA? A large explorative cross-sectional study. Ann Rheum Dis 2018;77:861-8.

36. El-Sherif HE, Kamal R, Moawyah O. Hand osteoarthritis and bone mineral density in postmenopausal women; clinical relevance to hand function, pain and disability. Osteoarthritis Cartilage 2008; 16:12-7.

37. Hochberg MC, Lethbridge-Cejku M, Tobin JD. Bone mineral density and osteoarthritis: Data from the Baltimore Longitudinal Study of Aging. Osteoarthritis Cartilage 2004;12 Suppl A:45-8.

38. Haara MM, Arokoski JPA, Kröger H, Kärkkäinen A, Manninen P, Knekt P, et al. Association of radiological hand osteoarthritis with bone mineral mass: a population study. Rheumatology 2005;44:1549-54.

39. Driban JB, Ward RJ, Eaton CB, Lo GH, Price LL, Lu B, et al. Meniscal extrusion or subchondral damage characterize incident accelerated osteoarthritis: data from the Osteoarthritis Initiative. Clin Anat 2015;28:792-9. 